This item was submitted to Loughborough's Research Repository by the author.

Items in Figshare are protected by copyright, with all rights reserved, unless otherwise indicated.

\title{
Investigation of the soot formation in ethylene laminar diffusion flames when diluted with helium or supplemented by hydrogen
}

PLEASE CITE THE PUBLISHED VERSION

http://dx.doi.org/10.1021/ef401970q

\section{PUBLISHER}

(c) American Chemical Society

\section{VERSION}

AM (Accepted Manuscript)

\section{PUBLISHER STATEMENT}

This work is made available according to the conditions of the Creative Commons Attribution-NonCommercialNoDerivatives 4.0 International (CC BY-NC-ND 4.0) licence. Full details of this licence are available at: https://creativecommons.org/licenses/by-nc-nd/4.0/

\section{LICENCE}

CC BY-NC-ND 4.0

\section{REPOSITORY RECORD}

Zhao, Huayong, Richard Stone, and Ben Williams. 2014. "Investigation of the Soot Formation in Ethylene Laminar Diffusion Flames When Diluted with Helium or Supplemented by Hydrogen". Loughborough University. https://hdl.handle.net/2134/20059. 


\title{
Investigation of the soot formation in ethylene laminar diffusion flames when diluted with helium or supplemented by hydrogen
}

\author{
Huayong Zhao ${ }^{\mathrm{a}, 1}$, Richard Stone ${ }^{\mathrm{b}}$, Ben Williams ${ }^{\mathrm{c}}$ \\ ${ }^{a}$ Wolfson School of Mechanical and Manufacturing Engineering, Loughborough University, Loughborough, LE11 $3 T U$ \\ ${ }^{b}$ Department of Engineering Science, University of Oxford, Parks Road, Oxford, U.K., OXI 3PJ \\ ${ }^{c}$ Department of Physics, University of Oxford, Parks Road, Oxford, U.K., OX13PU
}

\begin{abstract}
A new optical diagnostic technique has been used to measure the spatially distributed temperatures, soot diameters, and soot volume fractions in several different ethylene laminar diffusion flames to investigate the effect of adding hydrogen and helium on the soot formation. The test results show that adding hydrogen increases the flame temperature in all regions while adding helium does not significantly affect the flame temperature in the reaction region but does increase the flame temperature elsewhere. The flame heights when adding helium and hydrogen can be calculated by using the correlation introduced by Roper [4] if the ethylene diffusion coefficient is used. This indicates that the flame height is determined by the diffusion of ethylene molecules when the hydrogen fraction is below $20 \%$. It was also found that either adding helium or hydrogen does not significantly affect the soot diameter but does reduce the soot volume fraction. $20 \%$ of helium addition by volume was measured to reduce the total soot number by $19 \%$ while $20 \%$ of hydrogen addition reduced the total soot number by $23 \%$. In comparison, replacing the hydrocarbon with hydrogen is much more effective in reducing soot formation. Replacement of $25 \%$ ethylene by hydrogen was measured to reduce the total soot number by $66 \%$. Apart from demonstrating the influence of hydrogen and helium on ethylene diffusion flames, these measurements provide additional data for modellers of diffusion flames, especially those with an interest in the formation of particulate matter.
\end{abstract}

Keywords: soot formation; ethylene; hydrogen; helium; laminar diffusion flame

\section{Introduction}

Hydrogen has been considered as a strong candidate for the fuel of the future for a long time, because of its clean combustion and sustainability. However, it will take a long time to make the transition from conventional hydrocarbon based fuel combustion systems to pure hydrogen based combustion systems, due to the shortage of infrastructure to produce, store and transport the hydrogen, and the poor economic competitiveness of hydrogen combustion systems. A hybrid hydrogen-hydrocarbon combustion system provides an attractive option during this transition period because it exploits the advantageous properties of hydrogen, while requiring very few modifications to current hydrocarbon-based combustion systems.

Development of such a hybrid system is based on a clear understanding of the combustion and emission characteristics of hydrogen-hydrocarbon combustion systems, and this has been an active research area. Ilbas et al. [1] found that adding hydrogen can dramatically increase the flame speed and thus the burning velocity of a methane-air flame. They also reported that adding hydrogen can ease the ignition of the hydrocarbon mixture (due to the low ignition energy of the hydrogen) and extend the flammability limit of hydrocarbon fuels. Huang et al. [2] reported that adding hydrogen increases the laminar burning velocity of the natural gas-hydrogen flame. In addition, they reported that an increase in hydrogen fraction also increases the thermo-diffusive generated flame instability at a specific equivalence ratio. Choudhuri and Gollahalli [3] investigated the combustion and emission characteristics of the hydrogen -Natural Gas (NG) and $\mathrm{H}_{2}-\mathrm{C}_{3} \mathrm{H}_{8}$ laminar diffusion flames. They reported that the visible flame height was reduced when the fraction of hydrogen was increased and the percentage reduction in the flame height was well predicted by the correlation suggested

\footnotetext{
${ }^{1}$ Correspondence Author Tel: +44 (0)1509 227568 Email: H.Zhao2@lboro.ac.uk
} 
by Roper [4]. They also found that the radiative heat loss fraction, average soot loading and $\mathrm{CO}$ emissions increased while the $\mathrm{NO}_{\mathrm{x}}$ was reduced with an increase of $\mathrm{NG}$ or $\mathrm{C}_{3} \mathrm{H}_{8}$ concentration. Schug et al. [5] suggested that the flame height was only dependent on the fuel flow rate and not affected by the additives but that the diluent would reduce the

flame temperature. This was in contrast to Roper's correlation, which suggested that the flame height was a function of total fuel flow rate, diffusivity of the fuel and additive, and the flame temperature. Schug et al. [5] also reported that the $\mathrm{C} / \mathrm{H}$ ratio of the fuel cannot be used as a parameter to indicate sooting tendency. As for the effect of adding hydrogen on soot formation, Gulder et al. [6] have reported several comprehensive experiments that investigated the effect of adding hydrogen on soot formation in different hydrocarbon laminar diffusion flames. Their measurements of temperature (by Coherent Anti-Stokes Raman Spectroscopy, CARS) showed that either adding helium or hydrogen did not affect the temperature distributions. The average axial soot volume fraction distributions (measured by line-of-sight attenuation) in their paper showed that adding helium reduced the soot formation by purely a dilution effect and that adding hydrogen reduced the soot volume fraction more effectively by a combination of dilution and chemical inhibition effects. The numerical simulations by Guo et al. [7] showed that flame temperature increased when adding hydrogen. Tsolakis et al. [8] investigated the effect of adding hydrogen on particulate emissions from a dual fuel diesel engine and reported that the particulate numbers can be reduced by the use of reformed exhaust gas recirculation (hydrogen rich gas), but with no effect on the particle size and mass distributions.

In an attempt to bring clarity to the contradictory results presented above about the flame height and flame temperature of the diluted and hydrogen-supplemented hydrocarbon flames, two different groups of ethylene laminar diffusion flame experiments have been undertaken by adding five different fractions $(2 \%, 5 \%, 10 \%, 15 \%, 20 \%$ by volume $)$ of helium and hydrogen. The spatially resolved temperature, soot diameter and soot volume fraction distributions will be reported in this paper to show the quantitative effect of adding hydrogen on soot formation. In addition, the effect of replacing different fractions of ethylene by hydrogen on soot formation will also be reported. Apart from demonstrating the influence of hydrogen and helium in ethylene diffusion flames on temperature and soot loading distributions, these measurements provide additional data for modellers of diffusion flames, especially those with an interest in the formation of particulate matter.

\section{Experiment setup and methodology}

The overall experimental setup is shown in Fig. 1.

\section{Fig. 1 Schematic experimental set-up}

A Santoro co-flow burner [7] with a chimney was used to produce the laminar diffusion flames. The chimney wall has been black anodized to minimize glare and light reflections. The mass flow rates of air and fuel were controlled by two MKS ${ }^{\circledR}$ mass flow controllers (MFCs), which can be remotely controlled by an interface control box and a program written in LABVIEW. A premixing chamber filled with beads was used for the mixing of the primary fuel and secondary fuel or diluent. A Photron ${ }^{\circledR}$ FASTCAM 1024PCI high speed camera, which had been spectrally calibrated, was used to record the flame image. The maximum resolution is $1024 * 1024$ pixels with a $17 \mu \mathrm{m}$ pixel width. The image can be saved in the 'Bayer' format which records the colour channel value for each pixel without any on-board processing such as colour demosaicing. Throughout the experiments, the acquisition rate of the camera was set at 60 frames/second; the effective aperture was chosen as $f / 32$ to balance the effect of diffraction and other aberrations with the depth of field; the lens used was a Nikon $f=50 \mathrm{~mm}$ with a $2^{*}$ macro convertor; a LEE 281 filter was employed to attenuate red light, promoting better use of the dynamic range of all three colour channels in the camera. A half-cone pointer which can be inserted into the fuel tube was used to find the focal plane. The flow conditions for the helium-diluted ethylene flames, hydrogen-ethylene flames with constant ethylene flow rate and hydrogen-ethylene flames with constant total fuel flow rate are summarized in table 1 . The air flow rate was kept constant at $45 \mathrm{~L} / \mathrm{min}$ for all of the experiments.

Table 1 Summary of flow rate $(\mathrm{mL} / \mathrm{min})$ conditions

Cone-Beam Tomographic Three Colour Spectrometry (CBT-TCS) was used to measure the spatially distributed temperature, soot diameter and soot volume fractions inside the laminar diffusion flames. This technique is based on the measurement of the thermal radiation emitted from soot particles by a colour camera. The volumetric property fields are reconstructed by using Planck's law, Mie scattering theory and a 3D tomography technique. The 'diameter' measured by 
this technique is the diameter of the equivalent spherical particle which has the same light scattering characteristics as the soot aggregate present in the flame. This diameter can be correlated satisfactorily with the overall soot aggregate size. The detailed methodology has been introduced elsewhere [9].

Fig. 2 (from [9]) shows various views of a typical 2D slice in the central plane of the pure ethylene flame. The first subimage shows the spatial distribution of red pixel value (i.e. quantifying the red light contribution from each location, and measured in arbitrary digitisation units, or camera counts), as recovered from the reconstruction algorithm. (The absolute calibration of the camera sensor indicates that each camera count corresponds to $85.4 * 10^{-18} \mathrm{~J}$ of incident light.) Flame height data can be extracted from the red pixel value map, by finding the uppermost pixel to cross a threshold value from each flame condition. The remaining sub-images show distributions of temperature, soot diameter and soot volume fraction, respectively.

Fig. 2 Reconstructed red pixel value, temperature, soot diameter and soot volume fraction distributions in the central plane of the pure ethylene flame (regions between two white lines are a low signal region) [9]

These plots show unexpected noise and artefacts, viz. lumpy patterns and vertical stripes. Figure 3 (from [9]) shows typical reconstructed colour and colour ratio values as a function of radius at a representative height. This figure shows that the artefacts are introduced by the process of taking colour ratios, and that the problem is worst in regions where the signal is either very low or relatively uniform. This suggests that the dynamic range of the camera may not be large enough to distinguish the small light intensity differences. To remove these artefacts, spatial mean filters $(0.27 * 0.27 \mathrm{~mm})$ were applied to the data in these regions at the cost of reduced spatial resolution.

Fig. 3 Typical reconstructed radial colour and colour ratio distributions (in camera pixel count). (3*RG: tripled Redto-Green ratio; RB: Red-to-Blue ratio) [9]

In order to make comparisons between the measured flames, two kinds of diagram proved useful:

First, the temperature, soot diameter and soot volume fractions at three discrete heights $(1.5 \mathrm{~cm}$ above fuel exit - just below the middle of the flame; $2.5 \mathrm{~cm}$ - in the middle of the flame; $3.5 \mathrm{~cm}$ - just above the middle of the flame) were plotted as a function of radius.

Second, kernel density estimation (using the parameters listed in table 2) was applied to the 3D property fields to give the probability density distributions of temperature, soot diameter and soot volume fraction within the whole flame. The kernel used to smooth the discrete data was the Gaussian distribution with a bandwidth calculated according to the algorithm suggested in [10]. Because the soot volume fraction distribution in the central region (bounded by two white lines in fig. 2) suffers from a low signal-to-noise ratio, these data were discarded before analysis.

Table 2 Summary of the parameters used in the kernel density estimation process

As a final mode of comparison, the particle diameter $(d)$ and soot volume fraction $\left(f_{v}\right)$ data were used to calculate the total soot numbers $(N)$ inside the flames according to following equation:

$N=\sum \frac{6 * V_{v} * f_{v}}{\pi * d^{3}} \quad$ eq. (1)

Where $V_{v}$ is the voxel (volumetric pixel) volume.

Note that the soot volume fraction and diameter calculations are ill-conditioned compared to the temperature measurements, so temperature measurements might be extracted even when there is a lower signal to noise ratio. The signal to noise ratio is low in the central region of the flame when its diameter is large, and this is intrinsic to the tomographic reconstruction. The data in the central region are still presented in the radial property plots to illustrate trends, although the data quality is not as good as elsewhere.

Although no error bars are feature on the plots, the precision in the temperature measurement ranges from $1.5 \%$ to $3 \%$ and the precision in the soot volume fraction measurement ranges from $35 \%$ to $45 \%$ (see [9] where the sources of error are discussed in detail). The integrating effect of the kernel density estimation process makes it difficult to try and quantify the errors on these plots. 


\section{Results and discussion}

In this section, the effect of adding helium/hydrogen on the flame height, temperature and soot loading distributions are presented by comparing the radial distributions at three different heights and the kernel density estimations as previously described.

\subsection{Temperature}

Fig. 4 shows the radial flame temperature distributions at different flame heights when adding $20 \%$ helium and hydrogen. It shows that adding helium slightly increases the temperature below and above the middle part of the flame $(h=1.5 \mathrm{~cm}$ and $3.5 \mathrm{~cm})$ but does not affect the temperature in the middle part $(h=2.5 \mathrm{~cm})$. In comparison, adding hydrogen increases the flame temperature in all regions. To explain these observations, we propose that the temperature in the reaction zone is mainly controlled by chemical mechanisms. The middle part of the flame can be considered the most reactive region as temperature - and thus heat release - is highest there. As an inert gas, helium does not directly participate in these chemical reactions so its addition will only affect the temperature by the dilution effect, which means the helium will act as heat sink to reduce the reaction zone temperature. However, helium has a relatively low heat capacity, so its addition leads to a very small reduction in temperature, which is below the measurement precision. By contrast, hydrogen has a much higher calorific value $(120 \mathrm{MJ} / \mathrm{kg})$ than ethylene $(47.2 \mathrm{MJ} / \mathrm{kg})$ and actively participates in the chemical reactions so its addition increases heat release in the reaction zone. The increase in temperature outside the reaction zone when adding hydrogen or helium can be attributed to their high diffusion coefficients, which promotes heat transfer from the reaction zone. This plot also shows that adding helium slightly reduces the flame cross area in all heights (indicated by smaller radial range with measurable temperature value).

Fig. 4 Radial temperature distributions when at different heights $(h=1.5 \mathrm{~cm}$ is in the bottom part of the flame, $h=$ $2.5 \mathrm{~cm}$ is in the middle of the flame, $h=3.5 \mathrm{~cm}$ is in the top part of the flame; $\mathrm{He} 0$ - pure ethylene, $\mathrm{He} 20-$ adding $20 \%$ helium, H20 - adding 20\% hydrogen)

The kernel density estimations for the temperature, in Fig. 5, show more clearly that adding helium (a) or hydrogen (b) causes an increase in mean flame temperature, as the mean of the distribution increases with increasing addition in both cases. This effect is more pronounced when replacing a fraction of the ethylene with hydrogen, as can be seen in subplot (c) because of the combined effect of increased heat release during combustion and shorter flame (discussed in detail in section 3.2).

Fig. 5 Kernel density estimations of the temperature distributions when adding helium (a) (He10 - 10\%, He20 - 20\%) and hydrogen (b) (H10 - 10\%, H20 - 20\%) and replacing with hydrogen (c) (H10 - 10\%, H20 - 20\%)

\subsection{Flame height}

The height of a diffusion flame for a circular port burner can be calculated using the correlation introduced by Roper [4]:

$$
\frac{H}{Q}=\frac{1}{4 \pi D_{0}} \frac{1}{\ln \left(1+S^{-1}\right)}\left(\frac{T_{0}}{T_{f}}\right)^{0.67} \quad \text { eq. (1) }
$$

Where $H$ is the flame height; $Q$ is the total (combustible) fuel flow rate; $D_{0}$ is the initial diffusion coefficient; $S$ is the stoichiometric volume ratio of air to fuel; $T_{0}$ is the initial temperature; $T_{f}$ is the flame reaction region temperature.

Since helium does not participate in the chemical reactions in a flame, the flame height should not be affected by its addition, which is confirmed from the flame images. Considering the measurements of the ethylene-hydrogen flames, fig. 6 shows the measured flame heights and those calculated using the Roper correlation when adding and blending hydrogen. In this figure, two model lines are plotted, corresponding to the use of two different diffusion coefficients $\left(D_{0}\right)$ in eq. (1). The red solid line labelled 'Calc - Dx' employs a molar averaged diffusion coefficient:

$$
D_{x}=D_{0, \text { hydrogen }} * x+D_{0, \text { ethylene }} *(1-x) \quad \text { eq. }(2)
$$

Where $x$ is the molar fraction of hydrogen in the fuel mixture. The blue dashed curve labelled 'Calc - D' simply uses the diffusion coefficient of ethylene in air, $D_{0, \text { ethylene. }}$ 
Fig. 6 Comparison of flame heights measured by the camera (open circles) and calculated using Roper's correlation ('Calc - Dx': using molar averaged diffusion coefficient; 'Calc - D': using diffusion coefficient of ethylene)

Fig. 6 shows that ethylene-hydrogen flame height can be well predicted by Roper's correlation when the diffusion coefficient in eq. (1) is set to that of ethylene in air $\left(0.166 \mathrm{~cm}^{2} / \mathrm{s}\right)$. This suggests that flame height is determined by the diffusion of ethylene when the hydrogen fraction is below $20 \%$, despite the much higher diffusion coefficient in air $(0.61$ $\mathrm{cm}^{2} / \mathrm{s}$ ) of hydrogen.

\subsection{Soot diameters and soot volume fractions}

Fig. 7 shows the radial soot diameter distributions at three different heights when adding $20 \%$ helium and hydrogen. It shows that the particle size in the soot-loaded region (e.g. between $0.2-0.3 \mathrm{~cm}$ at $h=2.5 \mathrm{~cm}$ ) is largely unaffected by the addition of helium or hydrogen. The figure additionally shows that adding helium narrows the spatial extent of the soot-loaded region, possibly due to the dilution effect when adding helium reducing the oxygen rich region which is necessary for the pyrolysis reaction which forms soot nuclei. In contrast, adding hydrogen enlarges the soot-loaded region, potentially as the larger flammability range of hydrogen increases the size of the pyrolysis reaction region.

Fig. 7 Radial soot diameter distributions at different heights $(h=1.5 \mathrm{~cm}$ is in the bottom part of the flame, $h=2.5 \mathrm{~cm}$ is in the middle of the flame, $h=3.5 \mathrm{~cm}$ is in the top part of the flame; $\mathrm{He} 0$ - pure ethylene, $\mathrm{He} 20$ - adding $20 \%$ helium, H20 - adding 20\% hydrogen)

Fig. 8 shows the kernel density estimations of the soot diameters. It shows that either adding helium or hydrogen has no significant impact. But when replacing ethylene with hydrogen, the soot diameter distributions shift slightly towards smaller values, so more regions are covered by smaller diameter soot loadings. This might be due to the noticeably higher flame temperature when blending $20 \%$ hydrogen causing enhanced soot oxidation which reduces the number of large soot particles.

Fig. 8 Kernel density estimations of the soot diameter distributions when adding helium (a) (He10 - 10\%, He20 - 20\%) and hydrogen (b) (H10 - 10\%, H20 - 20\%) and replacing with hydrogen (c) (H10 - 10\%, H20 - 20\%)

The radial soot volume fraction distributions when adding $20 \%$ helium and hydrogen are shown in fig. 9. This shows that the typical result of adding helium or hydrogen is to reduce the measured soot volume fraction, particularly at the midflame height.

Fig. 9 Radial soot volume fraction distributions at different heights $(h=1.5 \mathrm{~cm}$ is in the bottom part of the flame, $h=$ $2.5 \mathrm{~cm}$ is in the middle of the flame, $h=3.5 \mathrm{~cm}$ is in the top part of the flame; $\mathrm{He} 0$ - pure ethylene, $\mathrm{He} 20$ adding 20\% helium, H20 - adding 20\% hydrogen)

The kernel density estimations for the soot volume fraction are plotted in fig. 10. This shows clearly that adding helium slightly reduces the presence of higher soot volume fraction (2-3 ppm) zones in the flame, while adding hydrogen is more effective. Replacing part of the ethylene with hydrogen produces a very marked change, with $20 \%$ replacement essentially eliminating any measurements of soot volume fraction above $\sim 1.3 \mathrm{ppm}$.

Fig. 10 Kernel density estimations of the soot diameter distributions when adding helium (a) (He10 - 10\%, He20 - 20\%) and hydrogen (b) (H10 - 10\%, H20 - 20\%) and replacing with hydrogen (c) (H10 - 10\%, H20 - 20\%)

Fig. 11 shows the total soot number when using different fuel mixtures. It clearly shows that either adding helium or hydrogen can reduce soot formation but that blending in hydrogen (i.e. replacing ethylene) is significantly more effective. The data shows that addition of $20 \%$ helium reduced the total soot number by $19 \%$, in line with dilution. Considering hydrogen instead, $20 \%$ addition reduced the total soot particle number by $23 \%$. The most dramatic reduction in soot formation occurs when replacing part of the ethylene with hydrogen, with a $25 \%$ hydrogen blend reducing the total soot number by $66 \%$.

Fig. 11 Total soot number when adding different fractions of helium/hydrogen ('Adding H2' means the ethylene flow rate is constant; 'Blending $\mathrm{H} 2$ ' means the total fuel flow rate is constant) 
The reduction in soot formation when adding helium matches expectations of the dilution effect, while the reduction when adding hydrogen is believed to be mainly a result of the direct chemical inhibition effect. According to the HACA model [11], the formation of soot precursor PAH (Polycyclic Aromatic Hydrocarbons) molecules can be simplified into two key steps: $\mathrm{H}$-abstraction and $\mathrm{C}_{2} \mathrm{H}_{2}$-addition, so the rate of formation depends on the concentrations of $\mathrm{C}_{2} \mathrm{H}_{2}, \mathrm{H}$ and $\mathrm{H}_{2}$. Considering these experiments, adding hydrogen increases the concentration of molecular hydrogen $\left(\mathrm{H}_{2}\right)$, which will increase the reverse reaction rate in the $\mathrm{H}$-abstraction reaction, closing reaction sites before $\mathrm{C}_{2} \mathrm{H}_{2}$ may bind. As a consequence, PAH molecules form more slowly and the number of available particle nuclei is reduced. In the next step of soot formation, larger particles are formed through coagulation and surface growth. The coagulation rate is proportional to the number concentration of the primary particles such that the coagulation rate will also be reduced when adding hydrogen. However, the higher flame temperature may promote the coagulation rate because of the increased kinetic energy available to the nuclei particles. According to the HACA surface reaction model [12], the surface growth rate is proportional to the acetylene concentration, the active surface site density and the particle surface area. Simulations undertaken by Guo et al. [7] indicate that adding hydrogen reduces the active surface site density but increases the acetylene concentration. Therefore, the reduced proportion of larger soot particles can also be attributed to the reduction in active surface sites. Overall, the dramatic reduction in soot formation when replacing part of the ethylene with hydrogen is due to this described chemical inhibition effect combined with the reduced number of carbon atoms.

\section{Conclusion}

In summary, the helium-ethylene-air laminar diffusion flame experiments show that adding helium does not affect the temperature distribution significantly, but does reduce the soot concentration $(20 \%$ of helium addition by volume can reduce the total soot number by 19\%), especially in the central section of the flame, and this is due to the dilution effect. However, adding helium slightly increases the extent of the high temperature region because the high diffusion coefficient of helium promotes heat transfer.

The hydrogen-ethylene-air flame experiments show that adding hydrogen has little effect on the flame height. This trend was predicted by the correlation suggested by Roper [4] providing the diffusion coefficient of ethylene in air is used as the overall diffusion coefficient. This result indicates that the flame height is determined by the diffusion of ethylene. Adding hydrogen increases the flame temperature because of the increased heat release. Adding hydrogen also reduces the soot concentration more effectively than adding helium due to the combined effect of dilution and direct chemical inhibition ( $20 \%$ of hydrogen addition can reduce the total soot number by $23 \%$ ). The primary chemical path disturbed by adding hydrogen is thought to be the increased reverse reaction rate of the H-abstraction reaction. In addition, adding hydrogen increases the proportion of the high temperature and low soot volume fraction region. This means adding hydrogen may also reduce the soot surface growth rate.

To investigate the effect of replacing hydrocarbon fuels with hydrogen on soot formation, different hydrogen-ethylene mixtures with a constant total fuel flow rate were used as the fuel. The results show that replacing hydrocarbons with hydrogen reduces the flame height. The correlation suggested by Roper can be used to successfully estimate the flame heights, by using the diffusion coefficient of ethylene alone, as before. Increasing the fraction of hydrogen can significantly reduce soot formation in laminar diffusion flames. Replacement of $25 \%$ ethylene by hydrogen was shown to reduce the total soot number by $66 \%$. This is not only due to the dilution and chemical inhibition effects, but is also due to the reduced number of carbon atoms.

\section{Reference}

[1] Ilbas, M; Crayford, A. P.; Yilmaz, I.; Bowen, P. J.; Syred, N. Int. J. Hydrogen Energy 2006, 31, 1768-1779.

[2] Huang, Z.; Zhang, Y.; Wang, Q.; Wang, J.; Jiang, D.; Miao, H. Energy \& Fuels 2006, 20, 2385-2390.

[3] Choudhuri, A. R.; Gollahalli, S. R. Int. J. Hydrogen Energy 2000, 25, 451-462.

[4] Roper, F. G. Combust. Flame 1977, 29, 219-226.

[5] Schug, K. P.; Manheimer-Timnat, Y.; Yaccarino, P.; Glassman, I. Combust. Sci. Technol. 1980, 22, $235-250$.

[6] Gulder, O. L.; Snelling, D. R., Sawchuk, R. A. Symposium (International) on Combustion, 1996, 2351-2358.

[7] Guo, H.; Liu, F.; Smallwood, G. J.; Gulder, O. L. Combust. Flame 2006, 145, 324-338.

[8] Tsolakis, A.; Hernandez, J. J.; Megaritis, A.; Crampton, M. Energy \& Fuels 2005, 19, 418-425. 
[9] Zhao, H.; Williams, B.; Stone, R. J. Quant. Spectrosc. Radiat. Transfer 2013. http://dx.doi.org/10.1016/ j.jqsrt.2013.07.024

[10] Botev, Z. I.; Grotowski, J. F.; Kroese, D. P. Ann. Statist., 2010; 38; 2916-2957.

[11] Frenklach, M.; Wang, H. Symposium (International) on Combustion, 1991, 23, 1559-1566.

[12] Appel, J.; Bockhorn, H.; Frenklach, M. Combust. Flame, 2000, 121, 122-136. 


\section{Tables}

Table 1 Summary of flow rate $(\mathrm{mL} / \mathrm{min})$ conditions

\begin{tabular}{lcccccc}
\hline Label & $\mathrm{He} 0$ & $\mathrm{He} 2$ & $\mathrm{He} 5$ & $\mathrm{He} 10$ & $\mathrm{He} 15$ & $\mathrm{He} 20$ \\
\hline$\% \mathrm{He}$ & 0 & 2 & 5 & 10 & 15 & 20 \\
$\mathrm{C}_{2} \mathrm{H}_{4}$ & 145 & 145 & 145 & 145 & 145 & 145 \\
$\mathrm{He}$ & 0 & 2.9 & 7.5 & 15.78 & 25.06 & 35.5 \\
\hline
\end{tabular}

(a) Helium-ethylene flame experiments

\begin{tabular}{lcccccc}
\hline Label & $\mathrm{H} 0$ & $\mathrm{H} 2$ & $\mathrm{H} 5$ & $\mathrm{H} 10$ & $\mathrm{H} 15$ & $\mathrm{H} 20$ \\
\hline$\% \mathrm{H}_{2}$ & 0 & 2 & 5 & 10 & 15 & 20 \\
$\mathrm{C}_{2} \mathrm{H}_{4}$ & 145 & 145 & 145 & 145 & 145 & 145 \\
$\mathrm{H}_{2}$ & 0 & 2.9 & 7.5 & 15.78 & 25.06 & 35.5
\end{tabular}

(b) Hydrogen-ethylene flame experiments with constant total ethylene flow rate

\begin{tabular}{lccccccc}
\hline Label & $\mathrm{H} 0$ & $\mathrm{H} 2$ & $\mathrm{H} 5$ & $\mathrm{H} 10$ & $\mathrm{H} 15$ & $\mathrm{H} 20$ & $\mathrm{H} 25$ \\
\hline$\% \mathrm{H}_{2}$ & 0 & 2 & 5 & 10 & 15 & 20 & 25 \\
$\mathrm{C}_{2} \mathrm{H}_{4}$ & 145 & 142.5 & 137.25 & 130.5 & 123.25 & 116 & 108.75 \\
$\mathrm{H}_{2}$ & 0 & 2.9 & 7.25 & 14.5 & 21.75 & 29 & 36.25 \\
\hline
\end{tabular}

(c) Hydrogen-ethylene flame experiments with constant total fuel flow rate

Table 2 Summary of the parameters used in the kernel density estimation process

\begin{tabular}{cccc}
\hline Parameters & Temperature $(\mathrm{K})$ & Diameter $(\mathrm{nm})$ & Soot volume fraction $(\mathrm{ppm})$ \\
\hline Kernel numbers & 64 & 32 & 32 \\
Minimum value & 1500 & 0 & 0 \\
Maximum value & $\sim 2200$ & $\sim 85$ & $\sim 5$ \\
Resolution & $\sim 10.9$ & $\sim 2.65$ & $\sim 0.156$ \\
\hline
\end{tabular}


Figures

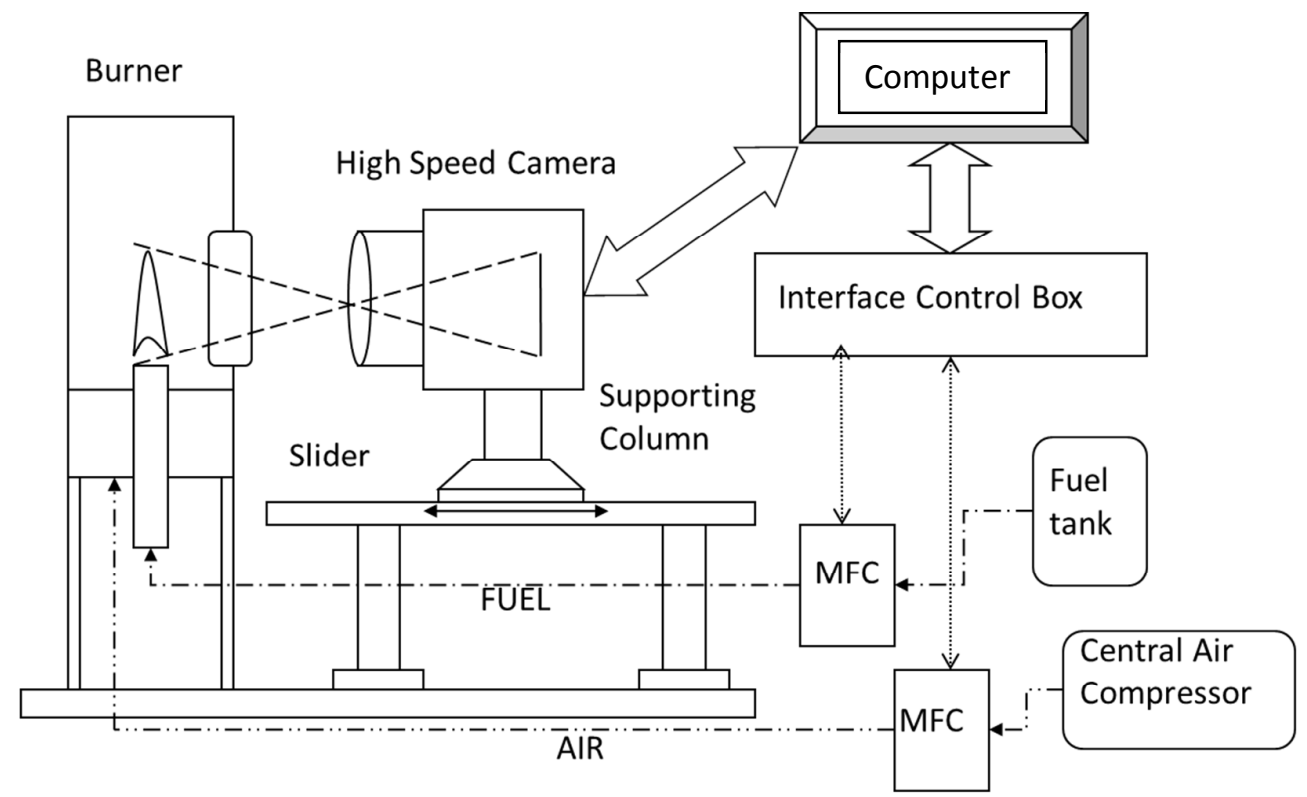

Fig. 1 Schematic experimental set-up. MFC stands for Mass Flow Controller.
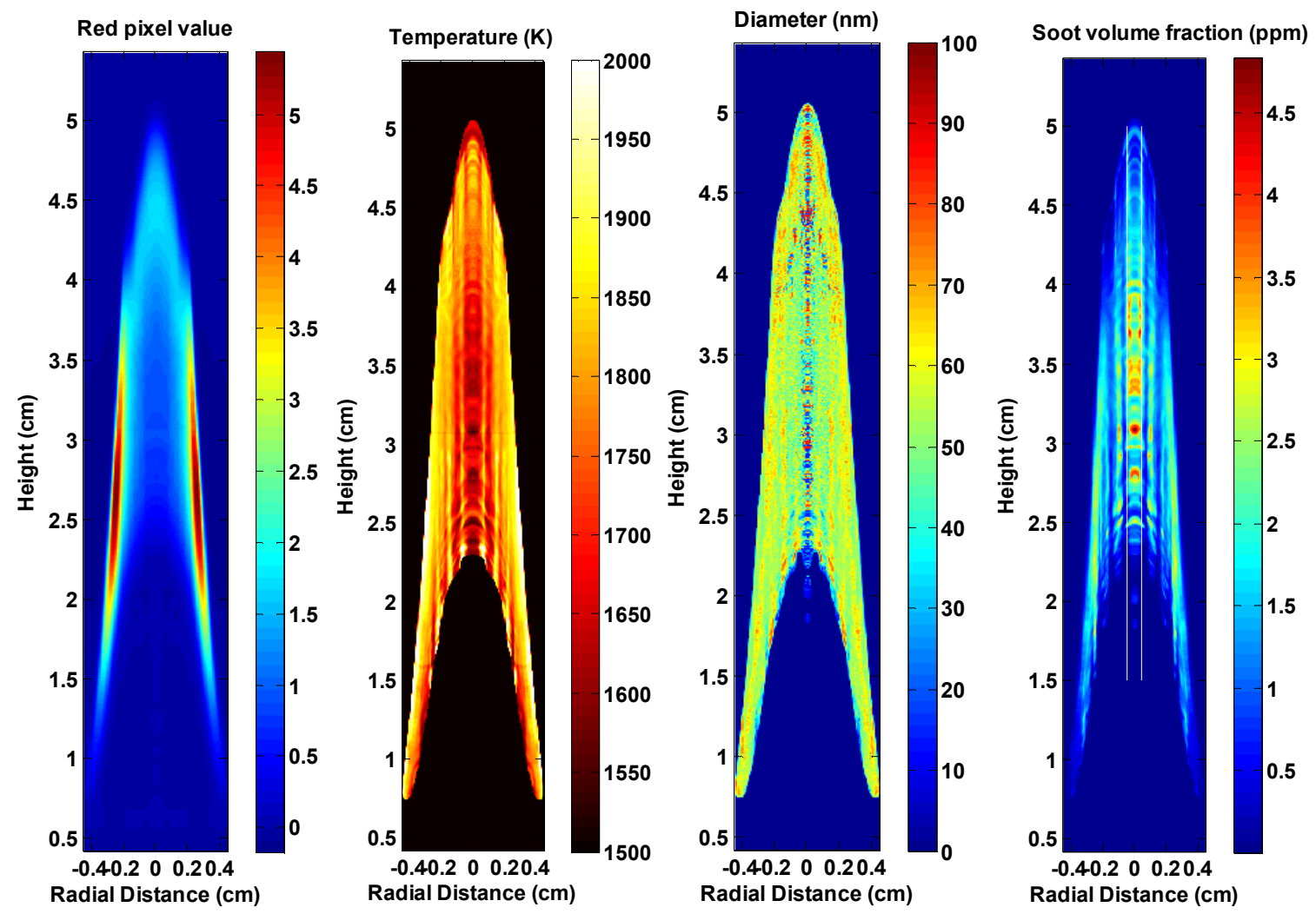

Fig. 2 Reconstructed red pixel value, temperature, soot diameter and soot volume fraction distributions in the central plane of the pure ethylene flame [9]. Vertical white lines denote boundaries of the low signal to noise region. For further details, see text. 


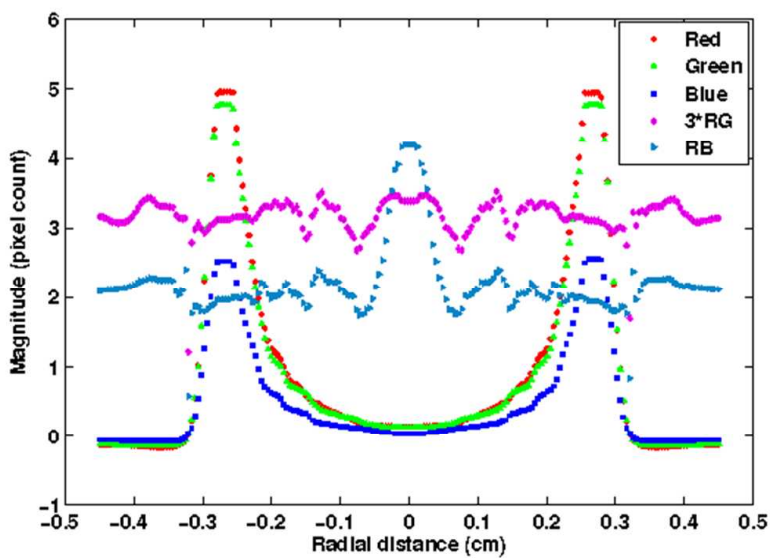

Fig. 3 Typical reconstructed radial colour and colour ratio distributions (in camera pixel count). ( $3 *$ RG: tripled Redto-Green ratio; RB: Red-to-Blue ratio) [9]
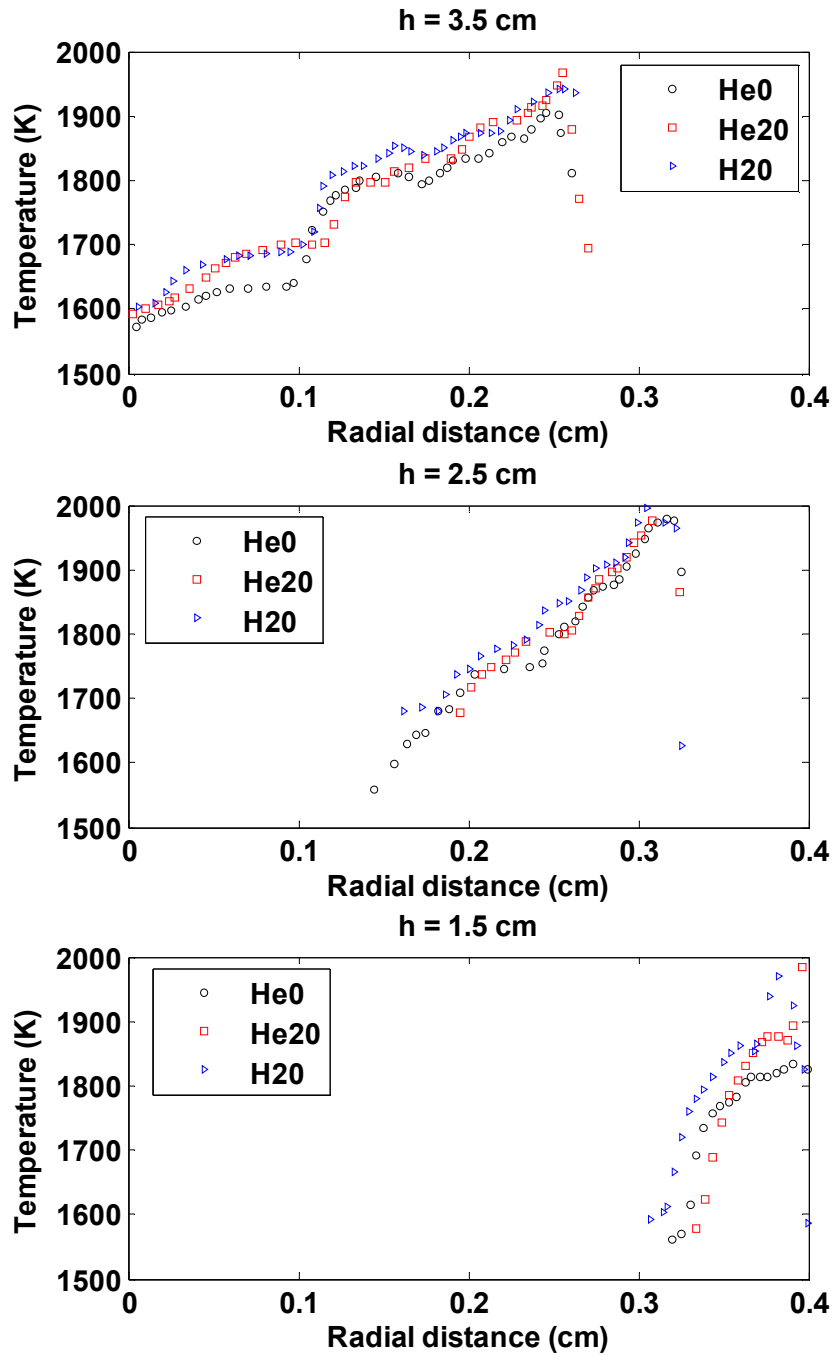

Fig. 4 Radial temperature distributions at different heights $(h=1.5 \mathrm{~cm}$ is in the bottom part of the flame, $h=2.5 \mathrm{~cm}$ is in the middle of the flame, $h=3.5 \mathrm{~cm}$ is in the top part of the flame; $\mathrm{He} 0$ - pure ethylene, $\mathrm{He} 20$ - adding $20 \%$ helium, H20 - adding 20\% hydrogen) 


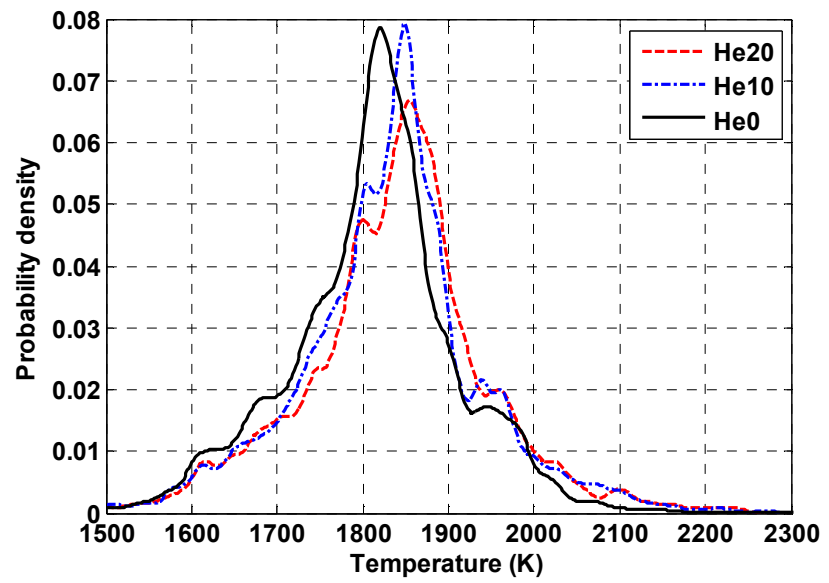

(a)

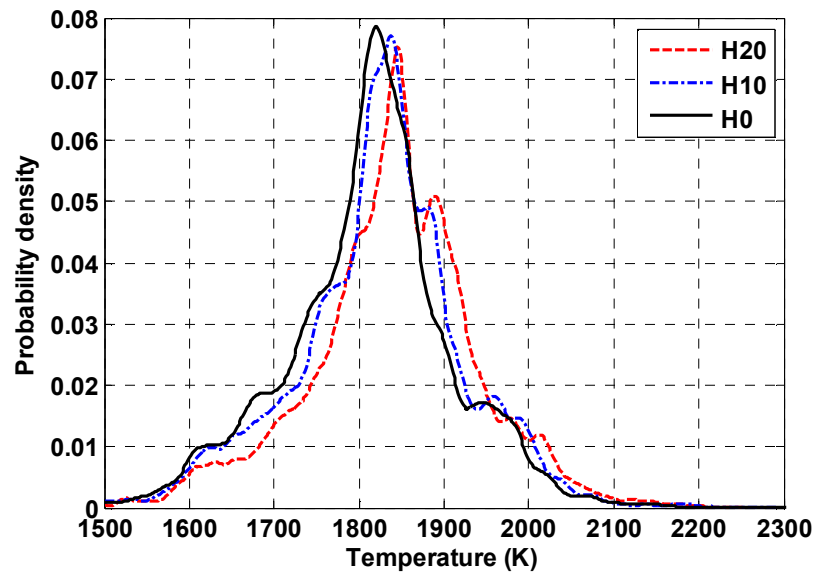

(b)

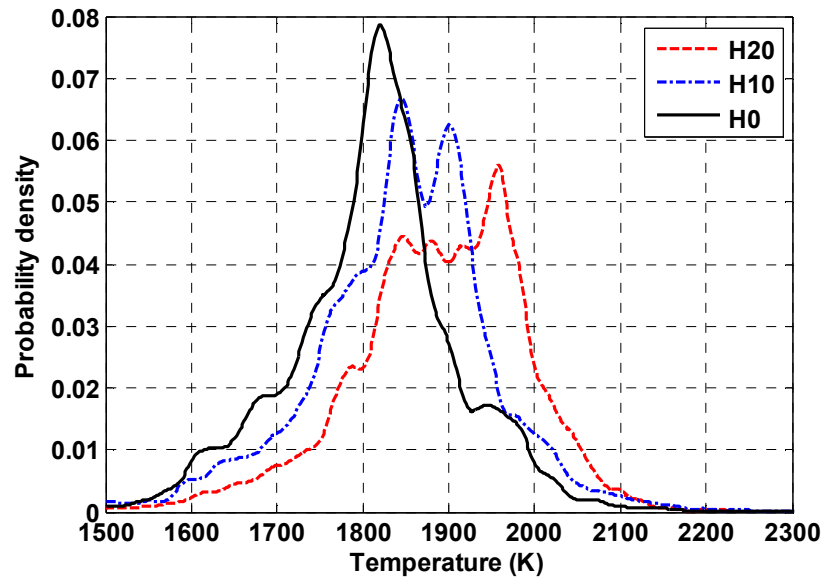

(c)

Fig. 5 Kernel density estimations of the temperature distributions when adding helium (a) (He10 - 10\%, He20 - 20\%) and hydrogen (b) (H10 - 10\%, H20 - 20\%) and replacing with hydrogen (c) ( $\mathrm{H} 10-10 \%, \mathrm{H} 20-20 \%)$ 

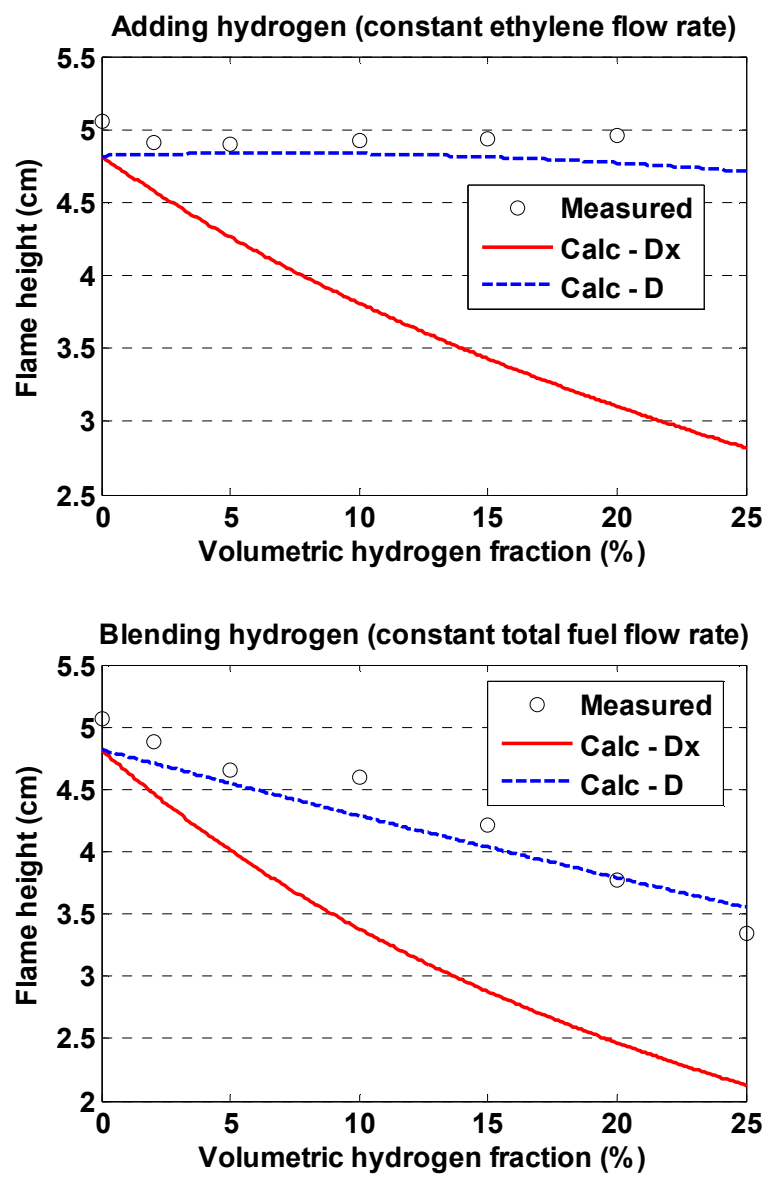

Fig. 6 Comparison of flame heights measured by the camera (open circles) and calculated by Roper's correlation ('Calc - Dx': using molar averaged diffusion coefficient; 'Calc - D': using diffusion coefficient of ethylene) 

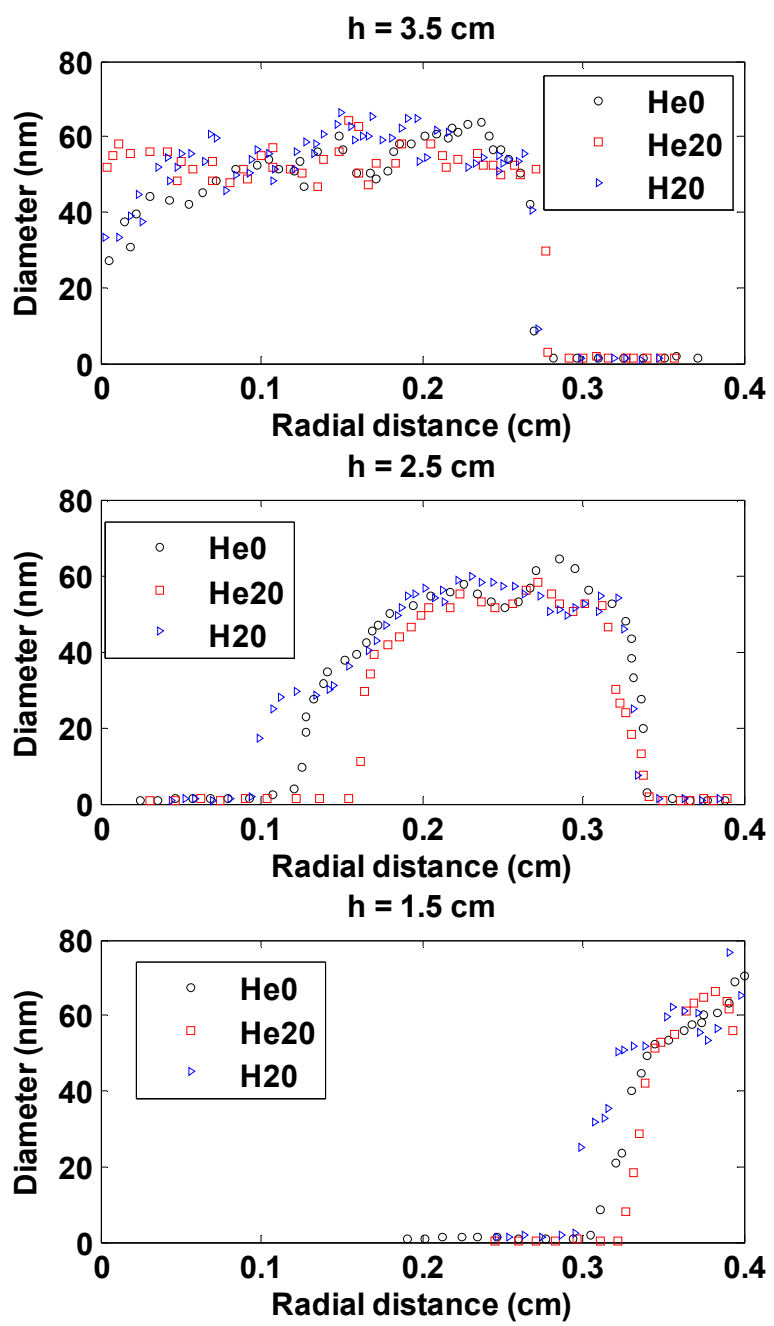

Fig. 7 Radial soot diameter distributions at different heights $(h=1.5 \mathrm{~cm}$ is in the bottom part of the flame, $h=2.5 \mathrm{~cm}$ is in the middle of the flame, $h=3.5 \mathrm{~cm}$ is in the top part of the flame; He 0 - pure ethylene, He20 - adding $20 \%$ helium, H20 - adding $20 \%$ hydrogen) 


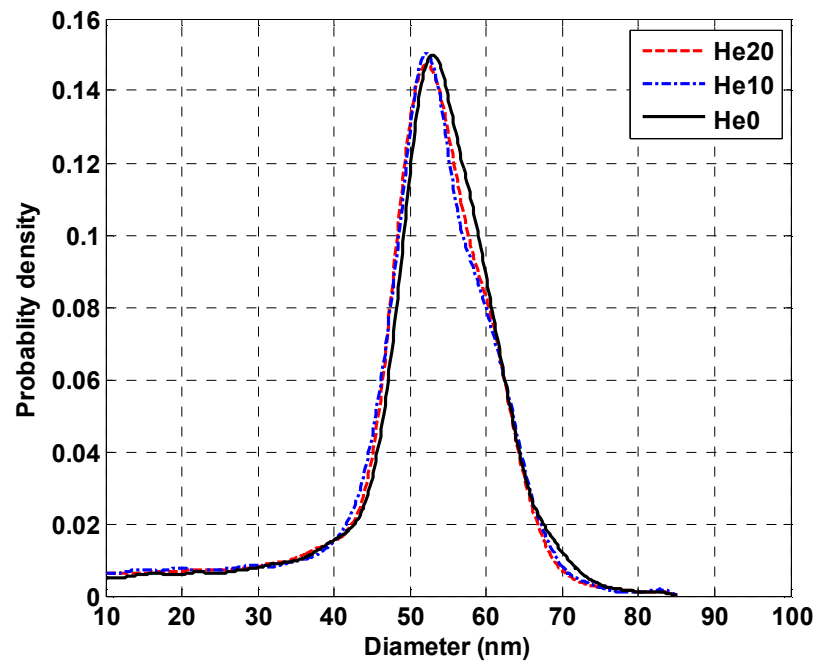

(a)

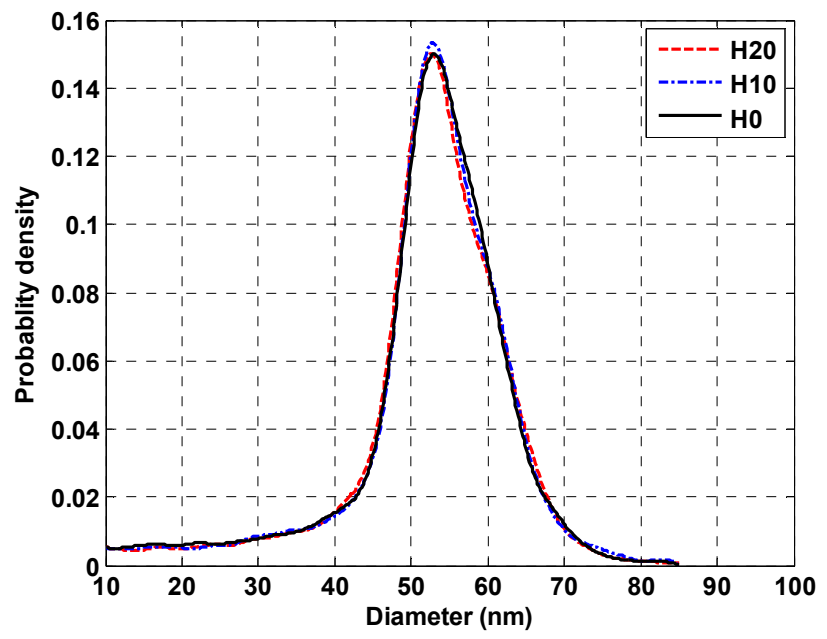

(b)

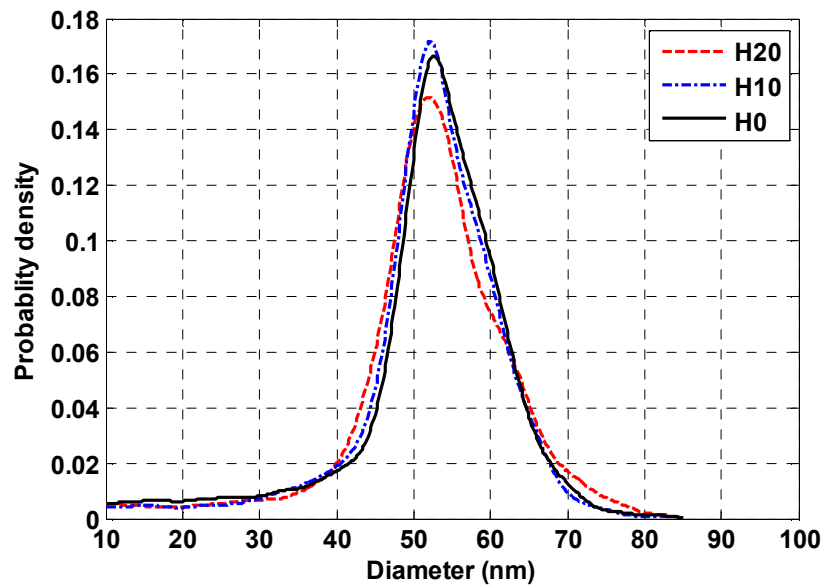

(c)

Fig. 8 Kernel density estimations of the soot diameter distributions when adding helium (a) (He10 - 10\%, He20 - 20\%) and hydrogen (b) (H10 - 10\%, $\mathrm{H} 20-20 \%)$ and replacing with hydrogen (c) (H10 - 10\%, $\mathrm{H} 20-20 \%)$ 

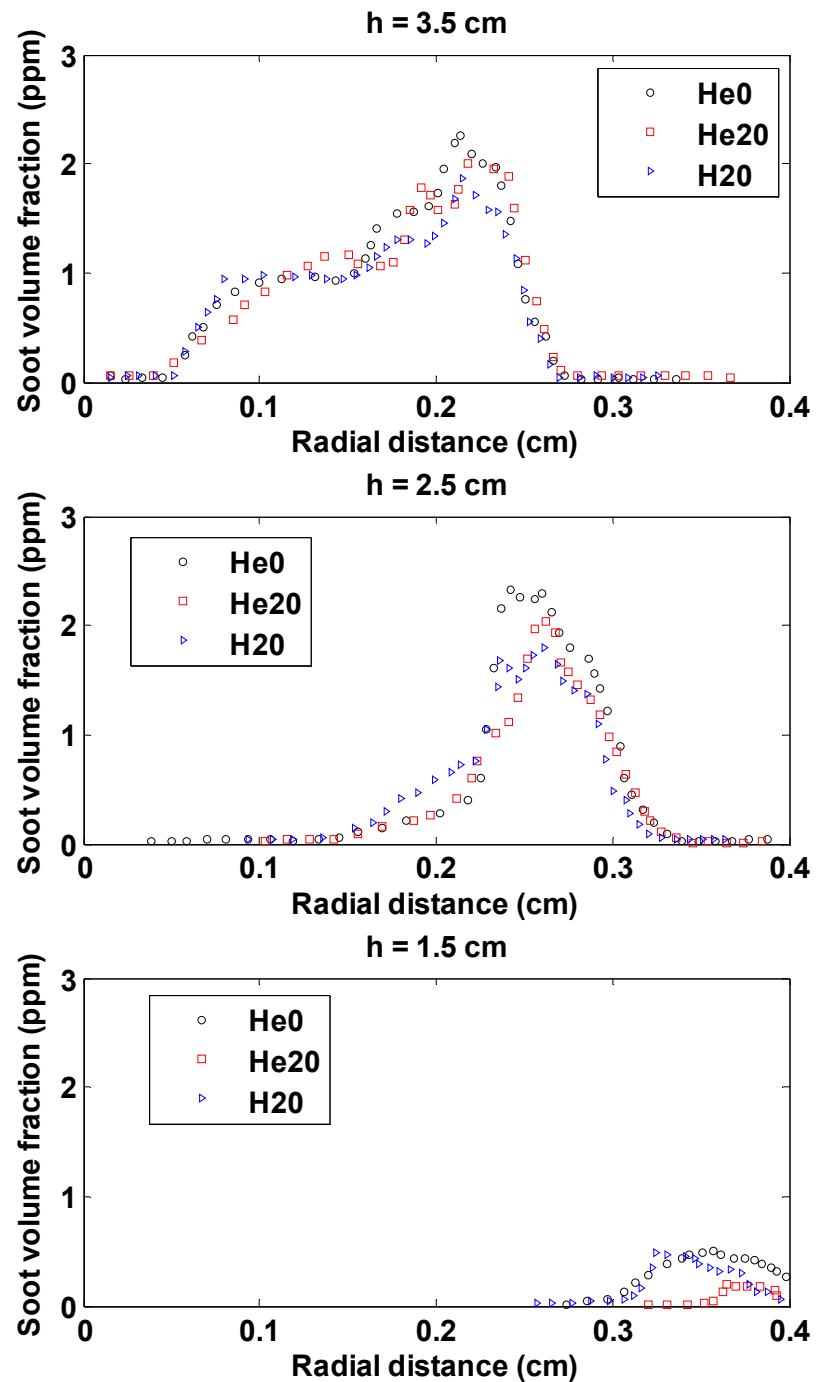

Fig. 9 Radial soot volume fraction distributions at different heights $(h=1.5 \mathrm{~cm}$ is in the bottom part of the flame, $h=$ $2.5 \mathrm{~cm}$ is in the middle of the flame, $h=3.5 \mathrm{~cm}$ is in the top part of the flame; $\mathrm{He} 0$ - pure ethylene, $\mathrm{He} 20$ adding $20 \%$ helium, H20 - adding 20\% hydrogen) 


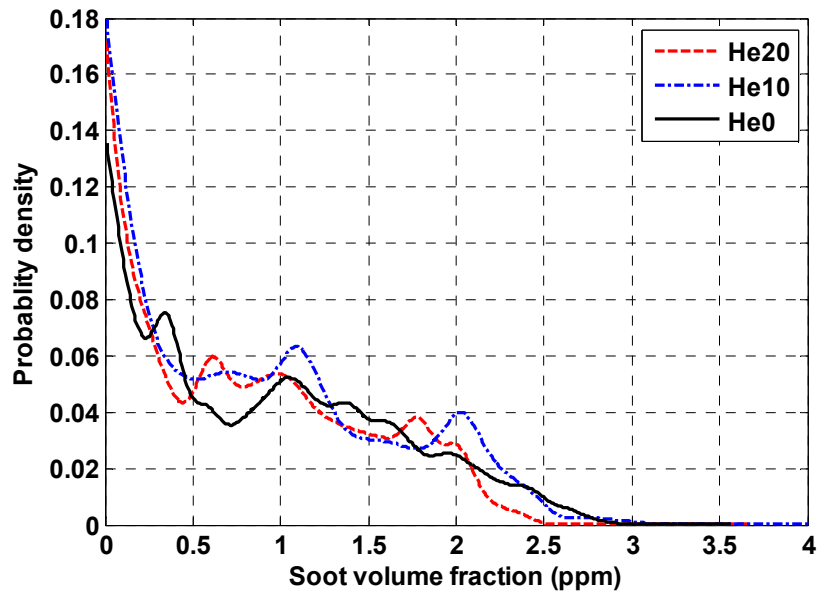

(a)

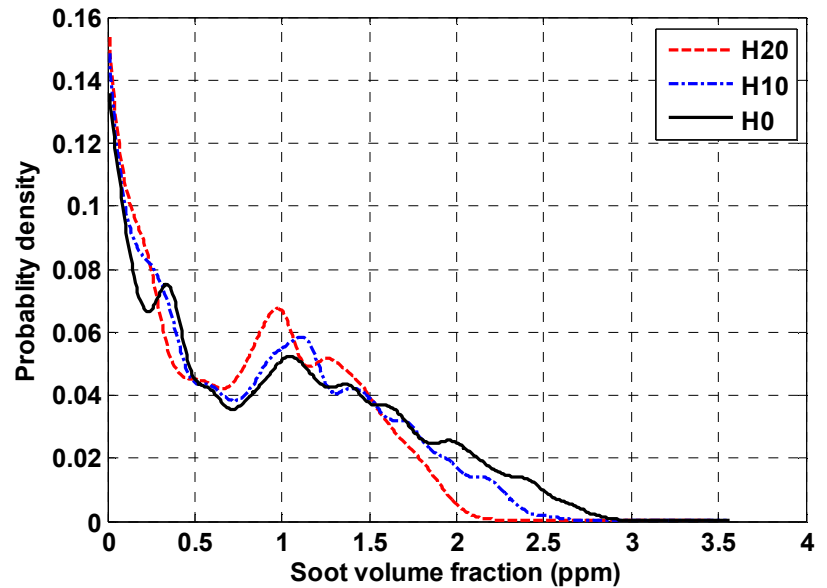

(b)

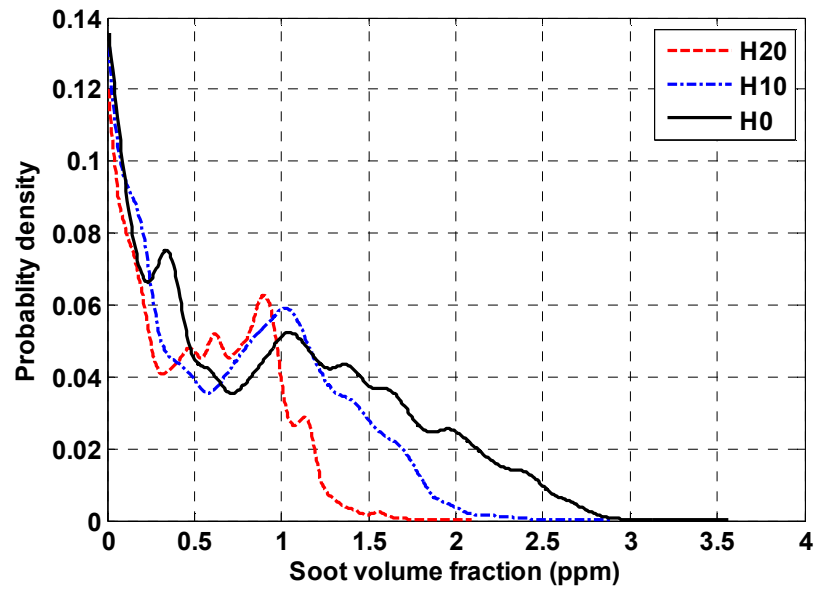

(c)

Fig. 10 Kernel density estimations of the temperature distributions when adding helium (a) (He10-10\%, He20 - 20\%) and hydrogen (b) (H10 - 10\%, H20 - 20\%) and replacing with hydrogen (c) (H10 - 10\%, H20-20\%) 


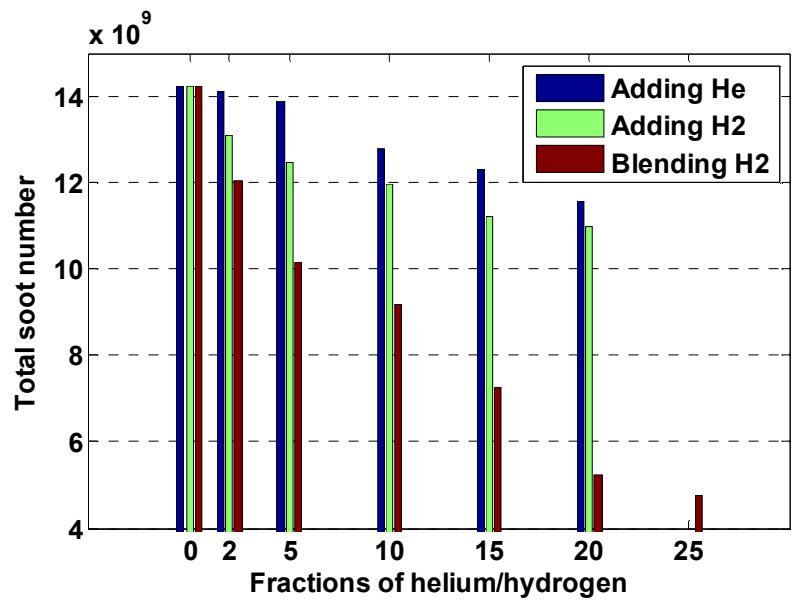

Fig. 11 Total soot number when adding different fractions of helium/hydrogen ('Adding H2' means the ethylene flow rate is constant; 'Blending H2' means the total fuel flow rate is constant) 